\title{
Antibodies: Basic Mechanisms and Emerging Concepts
}

\author{
Sudhir Gupta
}

Published online: 13 April 2010

(C) The Author(s) 2010. This article is published with open access at Springerlink.com

In recent years major advances in the understanding of the basic mechanisms of anti-inflammatory and immunomodulatory functions of antibodies have taken place. Immunoglobulin $\mathrm{G}$ is playing an ever-expanding role in the treatment of immunoinflammatory and neurologic disorders. Novel concepts in the pathogenesis of certain neurologic disorders and the possible role of natural antibodies and modulation by immunoglobulin therapy have emerged. These were discussed at the first International Forum on Immunoglobulin Research at Fort Lauderdale, FL, on November 19-22, 2009.

Intravenous immunoglobulin (IVIG) regulates immune responses by a number of complex mechanisms. These include its effects on inflammatory and immunomodulatory functions of dendritic cells (DCs; e.g., maturation of DCs, generation of Treg, and induction of tolerance) and the activation of different FcRs. Furthermore, different mechanisms of IVIG may predominate in different diseases, thus explaining its beneficial effects in a diverse group of disorders. Srini Kaveri (Paris, France) discussed the mechanisms of immunomodulatory effects of IVIG on DCs. However, it is critical to know the in vivo effects of IVIG on DC-T cell interactions. Studies of immunological synapses using twophoton microscopy should be able to define the in vivo effects of IVIG. Alan Lazarus (Toronto, Canada), in a murine ITP model using the adoptive transfer of IVIG-treated leukocytes or DCs, demonstrated that the therapeutic effect of IVIG may be mediated by specific effector cells. IVIG, following binding to DCs, upregulates the activation of $\mathrm{FcR}$, whereas in the recipient, inhibitory FcR appears to be necessary.

\section{S. Gupta $(\bowtie)$}

Division of Basic and Clinical Immunology,

University of California,

Irvine, CA, USA

e-mail:sgupta@uci.edu
Therefore, activated DCs may produce a factor that stimulates inhibitory FcR in the recipient.

Sialylation of IgG Fc plays an important role in the antiinflammatory effects of IVIG. In a $\mathrm{K} / \mathrm{BxN}$ serum-transfer model of arthritis, IVIG inhibited swelling and inflammatory cell infiltrate, and the removal of glycan or sialic acid abrogated the anti-inflammatory effects of IVIG. Robert Anthony (New York, NY, USA) and his group generated fully recombinant sialylated $\operatorname{IgG} 1 \mathrm{Fc}$ with enhanced potency. Marginal zone monocytes appear to be the target cells. FcRs are not required for sialylated Fc effects on macrophages. These sialylated Fcs bind to SIGN-R1 on macrophages, which produce a factor that in turn upregulates Fc $\gamma$ RIIb. The nature of the factor(s) remains to be determined. Genetic deletion of SIGN-R1 abrogates the anti-inflammatory effect of IVIG or sialylated Fc fragments. Friedrich Altmann (Vienna, Austria) presented structural requirements of IVIG (IgG) for binding to the lectin Sambucus nigra agglutinin (SNA). Twelve percent to $13 \%$ of IVIG bind to SNA. Binding of IVIG to the lectin was mediated by FAB glycosylation. Two sialic acids are required for SNA binding of glycan in the Fc region. This may suggest that double sialylation imposes changes of the protein conformation, which could be relevant for biological interactions. An interesting concept has emerged with regard to IgA FcR. Renato Monteiro (Paris, France) delineated the mechanisms whereby one Fc $\alpha \mathrm{R}-1$ could mediate both the anti-inflammatory properties of monomeric $\operatorname{IgA}$ and the proinflammatory properties of polymeric or aggregated IgA.

Intravenous immunoglobulin appears to have both proapoptotic and anti-apoptotic effects. Both of these effects target cell type and concentrations of IVIG may be determining factors. Hans-Uwe Simon (Bern, Switzerland) discussed the effects of IVIG on Fas-mediated apoptosis in 
PMN and eosinophils and demonstrated that low concentrations inhibit apoptosis, whereas high concentrations increased apoptosis (annexin V). In PMN, GM-CSF, and interferon- $\gamma$ increased the efficiency and potency of IVIGmediated apoptosis, whereas in eosinophils, IL-5 potentiated IVIG-induced apoptosis. The presence of anti-Siglec-9 autoantibodies appear to be responsible for chemokineaugmented cell death. Anti-Siglec antibodies are in dimeric form (anti-Siglec-anti-Id Siglec antibody). Neuraminidase treatment of IVIG has no effect. It remains to be determined whether these antibodies are natural antibodies. Furthermore, there is a need for in-depth analysis of apoptotic pathways (death receptor, mitochondrial, and the ER pathways of apoptosis) to understand underlying mechanisms of the beneficial effects of IVIG in certain disorders. This is particularly important with Alzheimer's disease because misfolded proteins trigger ER stress-induced apoptosis. Several IVIG clinical trials in $\mathrm{AD}$ are currently taking place.

Natural antibodies/autoantibodies (antibodies present before external antigen challenge) are produced by $\mathrm{B}_{1}$ cells and are made primarily from immunoglobulin genes without major somatic mutation. These antibodies are predominantly IgM and include a basic set of autoantibodies with low-affinity reactivity for multiple specificities; this set cross-reacts with common bacterial antigens, usually carbohydrate in nature. Natural antibodies may be antigen-specific; however, they are polyreactive. This may be due to the relative ubiquitous expression of target epitopes. However, epitopes recognized by natural antibodies remain to be characterized. Natural antibodies are predominantly IgM isotypes; IgG and IgA natural autoantibodies also occur. IgA natural autoantibodies are primarily found in secretory IgA. Natural antibodies (autoantibodies) provide three major functions: host defense, housekeeping, and immune homeostasis. The preliminary excitation of cells by autoantigens provides bacterial protection, especially because the polymeric nature of $\mathrm{CHO}$ antigens will mean that the IgM antibodies, although of low affinity, will bind with high avidity to the microbe (host defense). Natural antibodies may act as transporting agents that are responsible for scavenging effete body components (housekeeping). Natural antibodies also prevent the stimulation of autoreactive cells in the conventional $\mathrm{B}_{2}$ cell population either by masking autoantigen epitopes or by overall idiotypic regulation (homeostatic role).

More recently, the role and mechanisms of natural antibodies (autoantibodies) in the regulation of inflammation, allograft rejection, and remyelination of neuronal tissue have been delineated. Peter Lobo (Charlottesville, VA, USA) discussed a role of naturally occurring IgM antileukocyte antibodies (reacting with macrophages, PMN, and $\mathrm{T}$ cells). These antibodies, which are increased in acute inflammatory states, are of low binding affinity and kill leukocytes at cold temperature. Their physiological functions include protection from renal allograft and inhibition of $\mathrm{T}$ cell activation and cytokine production. These antibodies bind to $\mathrm{CD} 4 \mathrm{~T}$ cells. Recently, human $\operatorname{IgM~Fc}$ receptor $(\mathrm{F} c \mu \mathrm{R})$ has been identified. This receptor contains conserved Ser and Tyr residues in its cytoplasmic tail, but none of the Tyr residues match the immunoreceptor tyrosine-based activation, inhibitory, or switch motif. $\mathrm{Fc} \mu \mathrm{R}$ is expressed on $\mathrm{CD} 4^{+} \mathrm{T}$ cells and B cells. It is differentially regulated in that cross-linking results in the activation of $\mathrm{B}$ cells but in the inhibition of $\mathrm{T}$ cell activation. This may be helpful in understanding some of the mechanisms of natural IgM antibodies. Brian O'Nuallain (Dublin, Ireland) demonstrated that $\mathrm{A} \beta$-reactive natural $\mathrm{IgG}$ autoantibodies are present in almost all plasma and IVIG samples. These antibodies had minimal binding to $\mathrm{A} \beta$ monomers; however, they had good affinity for fibrils. Antibodies in IVIG crossreact with the conformational epitopes of $A \beta$ peptide. Sudhir Paul (Houston, TX, USA) discussed the properties of natural catalytic antibodies and reaction mechanisms involved in $\mathrm{A} \beta$ hydrolysis. Arthur Warrington (Rochester, NY, USA) presented data on the role of IgM natural antibodies in remyelination and data on the recombinant natural IgM antibody; however, the molecular characterization of target epitopes remains to be defined. Christoph Binder (Vienna, Austria) elegantly described a role of natural IgM antibodies in inflammation using an atherosclerosis model. This model is characterized by the accumulation of oxidized lipoproteins and apoptotic cells, which both contain various oxidation-specific neoepitopes. A majority of umbilical cord natural IgM antibodies react with these epitopes.

Intravenous immunoglobulin has been used in a variety of inflammatory and autoimmune neuropathies. Richard Hughes (London, UK) reviewed the incidence, prevalence, and healthcare costs for the treatment of chronic inflammatory demyelinating polyneuropathy (CIDP). He presented data from Cochrane Reviews, which suggest that IVIG, steroids, and plasma exchange are effective short-term therapies for CIDP. Clinical trial data also suggest that IVIG should be started at an initial dose of $2 \mathrm{~g} / \mathrm{kg}$ and followed by $1 \mathrm{~g} / \mathrm{kg}$ every 3 weeks; in addition, at least two doses should be given to decide whether a patient is a responder or nonresponder to IVIG. Jan Lünemann (Zurich, Switzerland) presented data that show the expression of Fc $\gamma$ RIIB (inhibitory receptor) on peripheral blood naive B cells in patients with CIDP is lower than in controls and fails to be upregulated as $\mathrm{B}$ cells progress from naive to the memory compartment. Furthermore, Fc $\gamma$ RIIB promoter polymorphism, which is associated with reduced promoter activity, was overrepresented in CIDP. Following IVIG therapy, Fc $\gamma$ RIIB expression on B cells and monocytes was 
upregulated and associated with a clinical response to IVIG. Pieter van Doorn (Rotterdam, The Netherlands) reviewed the clinical features and course of disease in Gullain-Barré syndrome and presented data on the efficacy of IVIG. He also identified that patients who had a minor increase in serum $\operatorname{IgG}$ levels following standard IVIG recovered significantly slower. Leonard van den Berg (Utrecht, The Netherlands) reviewed the clinical spectrum in multifocal motor neuropathy (MMN). He presented data of clinical outcomes following IVIG therapy, which highlighted the importance of early treatment to prevent axonal degeneration and more severe outcomes. His data also suggest that the effect of IVIG in MMN may at least be in part complement-mediated. Helen Chapel (Oxford, UK) discussed dosing, monitoring, compliance, and self-infusion of IVIG in MMN and CIDP.

Immune activation is necessary and sufficient to sustain experimental chronic pain. Spinal cord glial cell activation is critical to sustain sensory sensitization, and peripheral immune activation may contribute to such sensitization so that minor peripheral stimuli become painful in disorders such as fibromyalgia and complex regional pain syndrome (CRPS). Currently, randomized controlled trials of IVIG are ongoing in CRPS, painful peripheral neuropathy, and trigeminal neuralgia. Andreas Goebel (Liverpool, UK) reviewed various chronic pain syndromes and presented data from the recently completed randomized controlled trial on low-dose treatment in long-standing CRPS, which demonstrated good efficacy. IVIG has been effective in reducing pain intensity in patients with post-polio syndrome. He also reported the beneficial effect of both intrathecal and IVIG in an experimental model of spinal cord injury (SCI).

Autism is a heterogeneous multifactorial disorder with genetic, environmental, and immunological factors that appear to play a role in its pathogenesis. A number of immunological changes, which appear to be present in a subset of autistic children, include deficiency of $\mathrm{IgG}, \mathrm{IgG}$ subclasses and $\operatorname{IgA}$, and the presence of autoantibodies against neuronal antigens (Sudhir Gupta, Irvine, CA, USA). Two important features include increased inflammatory responses as evidenced by increased levels of chemokine and cytokine, both in the peripheral blood as well as in the CNS and tissue of the CNS, and the evidence of autoimmunity. Most interesting are the autism maternal antibodies against neural antigens. It is critical to define epitopes, which are targets of these antibodies, and then look at those epitope-specific antibodies in children with autism. Data on open-label trials of IVIG are conflicting: Beneficial effects are shown in 3 separate trials of 63 patients and 2 open trials of 15 patients ( 1 open trial had suboptimal dosing and 1 to 6 infusions at 6 weeks interval) that did not show any beneficial effect. Because of these conflicting data, it is important to plan a well-designed, double-blind study to determine the effectiveness of IVIG in autism. A good rationale for such a trial exists because of the disorder's association with antibody deficiency, autoimmunity, and inflammation.

Ion channels are essential for the basic physiological functions of excitable cells and immune cells. More than 340 human genes appear to encode ion channels, and mutations in more than 60 ion channel genes are known to cause human disorders known as channelopathies. Channelopathies, involving voltage-gated $\mathrm{Na}^{+}$channels, $\mathrm{Cl}^{-}$channels, voltage-gated $\mathrm{Ca}^{++}$channels, neuronal voltage-gated $\mathrm{K}^{+}$channels, inward rectified $\mathrm{K}^{+}$channels linked, and others, have been reported in a number of neuromuscular disorders. It also appears that some of the antibodymediated diseases of the nervous system may be due to antibodies directed against ion channels. Angela Vincent (Oxford, UK) gave an excellent description of various channelopathies in which serum antibodies are directed against voltage-gated $\mathrm{K}^{+}$channels or ligand-gated channels such as NMDA, AMPA, and glycine receptors. Some of the $\mathrm{K}^{+}$channel antibodies are directed against closely related proteins such as CASPR2 (a cell surface adhesion molecule). Antibody levels in the CSF are lower than in the serum; however, they are synthesized in the brain. Some of these disorders responded to IVIG, plasma exchange, and steroid treatment. During SCI, primary injury is a result of mechanical trauma, whereas secondary injury is caused by neuroinflammation as a result of the secretion of a number of inflammatory molecules, including pro-inflammatory cytokines and reactive oxygen species by locally recruited leukocytes and microglia. Michael Fehlings (Toronto, Canada) presented data from an SCI model in rats in which he demonstrated that IgG treatment reduces secondary inflammatory damage and improves hind limb function. This provided a rationale to investigate a neuroprotective role of Ig therapy in SCI.

Taking into consideration the role of natural IgM antibodies/autoantibodies and monomeric IgA in the inhibition of inflammation and autoimmunity, it is prudent to explore a possibility of treating some of these disorders with plasma preparations enriched in IgM or IgA. The definition of human IgM FcR may help in understanding the basic physiological mechanisms and mechanisms for the role of IgM natural antibodies in certain disorders. It is anticipated that as we continue to learn more about the mechanisms of action of immunoglobulins, their therapeutic use will expand into greater administration by different routes.

Open Access This article is distributed under the terms of the Creative Commons Attribution Noncommercial License which permits any noncommercial use, distribution, and reproduction in any medium, provided the original author(s) and source are credited. 